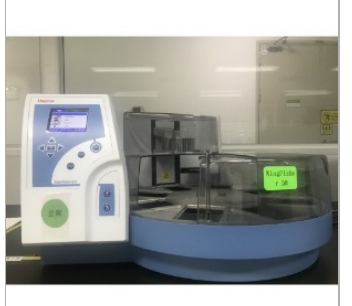

MAY 17, 2018

\title{
(3) DNA Extraction for plant samples by CTAB
}

Ting Yang ${ }^{1}$, Chenyu $\mathrm{Wu}^{1}$

${ }^{1}$ BGI-Shenzhen

GigaScience Press BGI

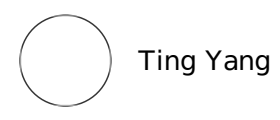

\section{ABSTRACT}

DNA extraction of $-80^{\circ} \mathrm{C}$ stored leaves by CTAB.

\section{MATERIALS}

MATERIALS

8 PVP Merck MilliporeSigma (Sigma-Aldrich)

$\$ 20$ mM EDTA Merck MilliporeSigma (Sigma-Aldrich)

88100 mM Tris-HCl Merck MilliporeSigma (Sigma-Aldrich)

$881.5 \mathrm{M} \mathrm{NaCl}$ Merck MilliporeSigma (Sigma-Aldrich)

$82 \%$ CTAB Merck MilliporeSigma (Sigma-Aldrich)

$\$ 1 \%$-mercaptoethanol BBI Biotech

$\$$ Chloroform: Isoamyl alcohol Sangon Biotech

8 isopropanol Sangon Biotech

$\$ 8$ sodium acetate Qiagen

8 ethanol BBI Biotech

88 TE buffer Thermo Fisher Scientific

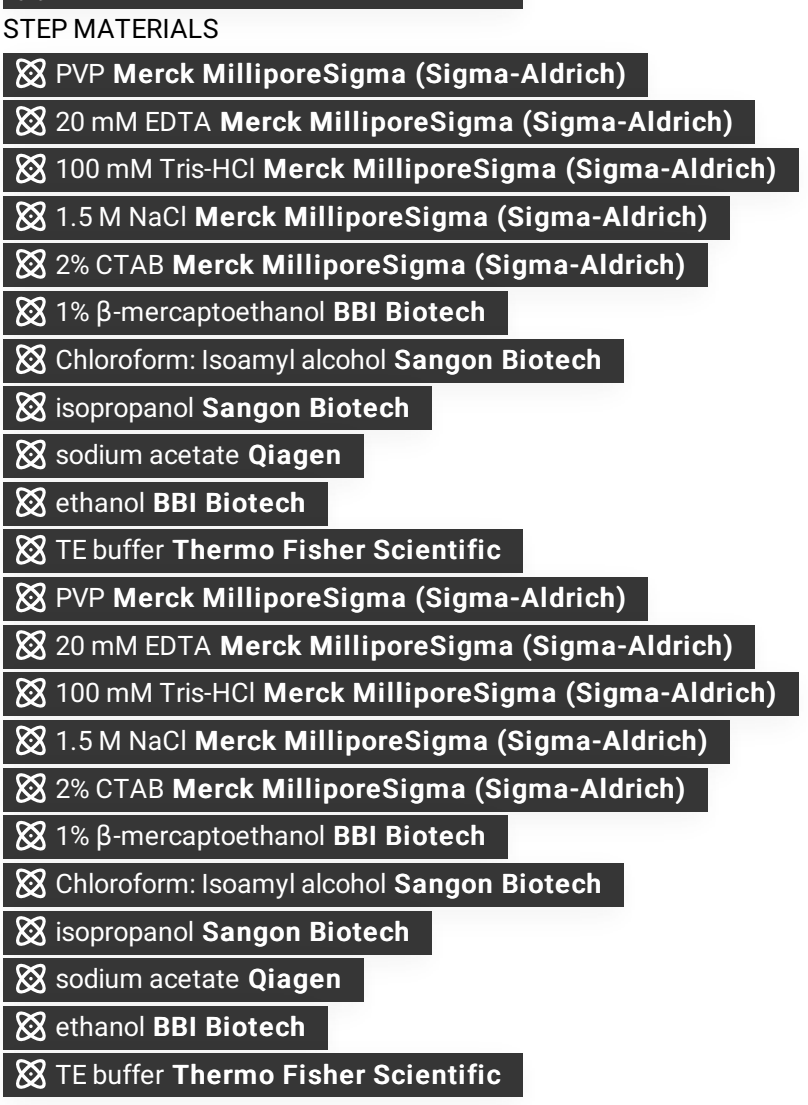

1 Grind $-80 \circ \mathrm{C}$ stored leaves (100-200 mg) to fine powder using liquid nitrogen in the presence of $50 \mathrm{mg}$ PVP (PolyVinyl Pyrrolidone, Mr 10,000). Note: It' s very important that the tissue doesn't thaw. 


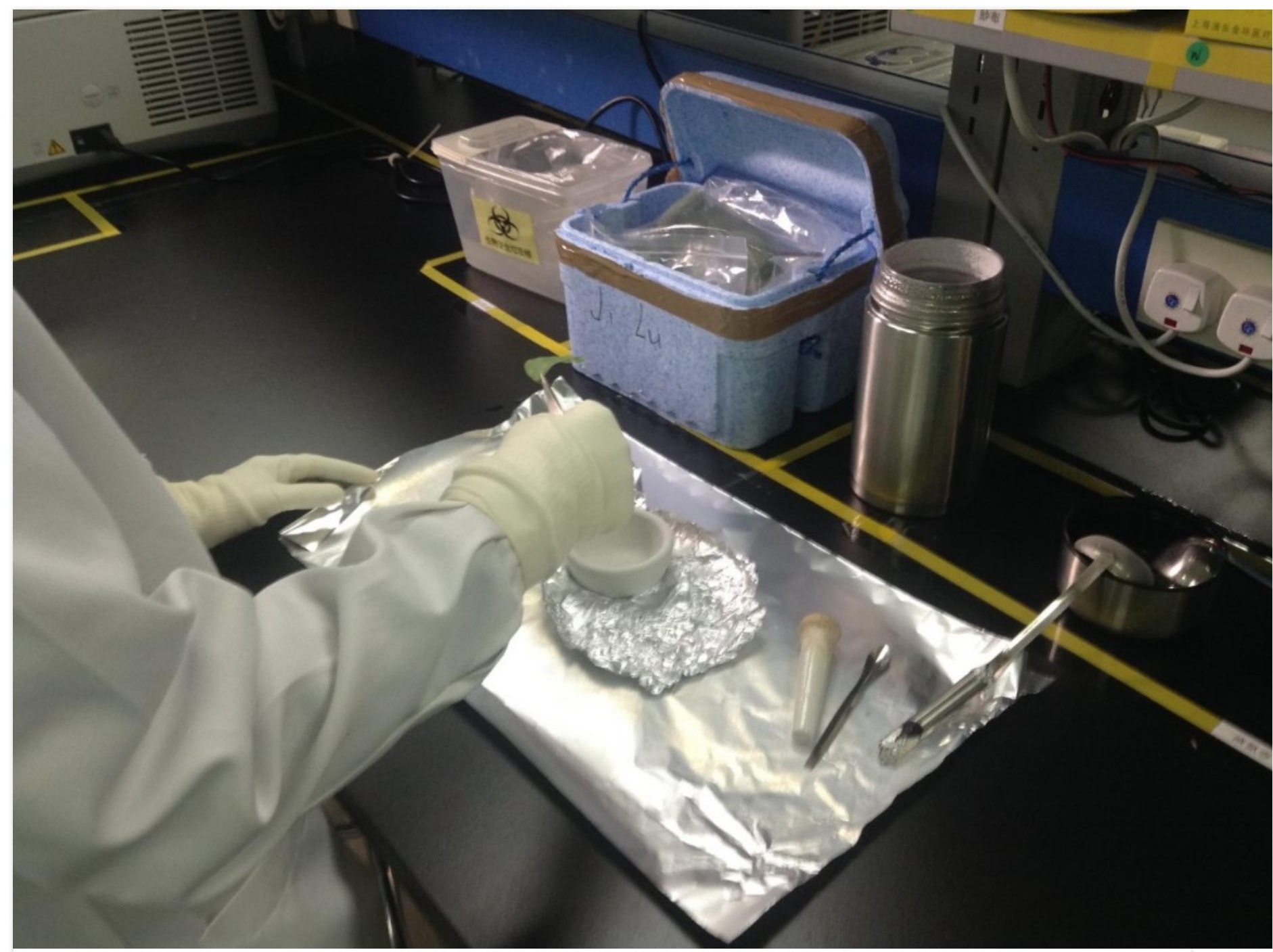

88 PVP Merck MilliporeSigma (Sigma-Aldrich)

2 Add $1.5 \mathrm{~mL}$ of freshly prepared $2 x \mathrm{CTAB}$ buffer and incubate at $60{ }^{\circ} \mathrm{C}$ for $45 \mathrm{~min}$ with intermittent shaking every 10 minutes.

$\begin{array}{lc}20 \mathrm{mM} \text { EDTA (PH 8.0, 0.5M) } & 40 \mathrm{ml} \\ 100 \mathrm{mM} \text { Tris- } \mathrm{HCl}(\mathrm{PH} 8.0,1 \mathrm{M}) & 100 \mathrm{ml} \\ 1.5 \mathrm{M} \mathrm{NaCl} & 87.6 \mathrm{~g} \\ 2 \% \mathrm{CTAB}(\mathrm{w} / \mathrm{v}) & 20 \mathrm{~g} \\ 1 \% \beta \text {-mercaptoethanol } & 10 \mathrm{ml} \text { (add before use) }\end{array}$

Then add distilled water to make it up to $1000 \mathrm{ml}$.

\section{0:45:00}

$\$ 20$ mM EDTA Merck MilliporeSigma (Sigma-Aldrich)

$\$ 8100$ mM Tris-HCl Merck MilliporeSigma (Sigma-Aldrich)

$81.5 \mathrm{M} \mathrm{NaCl}$ Merck MilliporeSigma (Sigma-Aldrich)

$82 \%$ CTAB Merck MilliporeSigma (Sigma-Aldrich)

$\$ 81 \%$-mercaptoethanol BBI Biotech

3 Centrifuge at $12,000 \mathrm{rpm}$ for $15 \mathrm{~min}$ at room temperature.

(3) 00:15:00

4 Carefully transfer the aqueous phase into a new tube. Note: Use wide-bore tips for transferring the aqueous phase to avoid mechanical damage to 
DNA.

5 Add double volume of Chloroform: Isoamyl alcohol (24:1) under fume hood, and invert gently 15 to 20 times and centrifuge at $12,500 \mathrm{rpm}$ for 15 $\min$. Note: If the aqueous layer appears translucent, repeat the stepuntil the solution is transparent. Be careful to avoid transferring any chloroform.

\section{0:15:00}

\section{Chloroform: Isoamyl alcohol Sangon Biotech}

6 Add double volume of chilled isopropanol followed by $1 / 3$ rd volume of $3 \mathrm{M}$ sodium acetate (pH 5.2) and keep at $-20 \circ \mathrm{C}$ for one hour to precipitate the DNA. Note: The longer the chilled incubation, the more the precipitation.

\section{1:00:00}

88 isopropanol Sangon Biotech

88 sodium acetate Qiagen

7 Centrifuge at $12,000 \mathrm{rpm}$ for $15 \mathrm{~min}$ and discard the supernatant.

\section{0:15:00}

8 To the pellet, add $70 \%$ chilled ethanol and spool out the pellet carefully and centrifuge again at 12,000 rpm for 15 min, and repeat the step again.

\section{0:15:00}

88 ethanol BBI Biotech

9 Discard the supernatant and vacuum dry or air dry the pellet at room temperature. Note: Make sure that there is no residual ethanol, this is very critical especially if the DNA is to be used directly for PCR. Overdrying should also be avoided as it makes the pellet difficult to resuspend.

10 Add 40-50 $\mu \mathrm{L}$ TE buffer ( $10 \mathrm{mM}$ Tris-HCl, $1 \mathrm{mM} \mathrm{EDTA}$, pH 8), and quantify the DNA using Qubit flurometer/Nanodrop, and run an $0.8 \%$ Agarose gel electrophoresis.

\section{TE buffer Thermo Fisher Scientific}

